solves problems which have already been solved for the Lorentz-Dirac equation. This weakens the raison d'être for their theory, and seems to sug- gest that they were too hasty in suggesting that their formalism replace the Lorentz-Dirac formalism.

\footnotetext{
*Research supported by the National Research Council of Canada under Grant No. A6286.

${ }^{1}$ T. C. Mo and C. H. Papas, Phys. Rev. D $\underline{4}, 3566$ (1971).

${ }^{2}$ F. Rohrlich, Classical Charged Particles (AddisonWesley, Reading, Mass., 1965).

${ }^{3}$ The conventional association is via Noether's theorem. No extremum is required, but only a vanishing first variation and invariance under a Lie group (the Poincaré group in this case). Also other derivations have been given within the context of new field-theoretic points of view [see Ref. 2, and also D. Leiter, Ann. Phys. (N.Y.) 51, 561 (1969); Lett. Nuovo Cimento $\underline{3}, 347$ (1970);
}

\begin{abstract}
Proc. Phys. Soc. London (Gen.) A3, 89 (1969)].
${ }^{4}$ See the section on the integral form of the LorentzDirac equation, and the associated boundary condition which precludes runaway solutions, in Ref. 2 .

${ }^{5}$ See J. D. Jackson, Classical Electrodynamics (Wiley, New York, 1962) for a good discussion of this point (e.g., chap. 17). The preacceleration effects are confined to times on the order of $10^{-23} \mathrm{sec}$. This is clearly within the quantum domain, and hence the predictions of classical mechanics are breaking down long before the effects can occur. For this reason they are not classically observable.
\end{abstract}

\title{
Reply to Comment on the "New Equation of Motion for Classical Charged Particles"
}

\author{
Tse Chin Mo and C. H. Papas \\ Electrical Engineering Department, California Institute of Technology, Pasadena, California 91109 \\ (Received 10 July 1972)
}

\begin{abstract}
We reply to a criticism made on the new equation of motion proposed by us for classical charged particles.
\end{abstract}

A new equation of motion for classical charged particles ${ }^{1}$ was proposed by us to account for radiation reaction. ${ }^{2}$ Here we reply briefly to a criticism of it made by Leiter ${ }^{3}$ and invite attention to the following.

(1) That we did not find an action principle for the new equation was explicitly pointed out in our original paper. The existence or nonexistence of such an action principle is still an open question.

(2) The validity or nonvalidity of an equation of motion is not determined by the existence or nonexistence of associated action principle (at least we believe so).

(3) The "validity" arguments for an "action principle" of the Lorentz-Dirac equation ${ }^{4}$ are already ample in the literature. ${ }^{5}$ Acknowledging these, Ref. 1 pointed out, just as a side remark, the contrary view that this "action principle" is not a physically valid action principle at all. We empha- size that this has no essential bearing on the proposed new equation.

(4) The energy-momentum conservation law required by and found for the new equation is "local" and is "nonlocal." It is "local" in the sense of being a pointwise differential relation, which says that something must happen in between if energymomentum decreases and increases at two places, and in the sense of the $t^{\mu \nu}$ being retarded from the sources. ${ }^{7}$ It is nonlocal in the sense that at each space-time point $x, T_{\mathrm{em}}^{\mu \nu}$ is not entirely made of the field $F^{\mu \nu}(x)$ at that point but has an additional piece $t^{\mu \nu}(x) .^{7}$ This was also pointed out in our original paper (Ref. 1).

(5) The runaway and preacceleration phenomena of the Lorentz-Dirac equation, which are well known and usually considered "solved,"" are exactly what dissatisfied and motivated us. 
${ }^{1}$ T. C. Mo and C. H. Papas, Phys. Rev. D $\underline{4}, 3566$ (1971).

${ }^{2} \mathrm{~A}$ further detail on the testability of the new equation is to be published elsewhere.

${ }^{3}$ D. Leiter, preceding paper, Phys. Rev. D $\underline{6}, 2292$ (1972).

${ }^{4}$ P. A. M. Dirac, Proc. Roy. Soc. (London) $\bar{A} 167,148$ (1938).

${ }^{5}$ See, e.g., F. Rohrlich, Phys. Rev. Letters 12,375 (1964).
${ }^{6}$ See R. P. Feynman, The Feynman Lectures on Physics (Addison-Wesley, Reading, Mass., 1964), Sec. 27-1 of Vol. 2 and Sec. 21-2 of Vol. 3.

${ }^{7}$ Ref. 1, Appendix.

${ }^{8}$ See any text treating classical radiation for the preacceleration including the integral-equation form of the Lorentz-Dirac equation, e.g., J. D. Jackson, Classical Electrodynamics (Wiley, New York, 1962), p. 597.

\title{
Comment on the Breakdown of Dilatation Invariance*
}

\author{
Antonio Aurilia and Yasushi Takahashi \\ Theoretical Physics Institute, University of Alberta, Edmonton, Alberta, Canada
}

(Received 22 May 1972)

\begin{abstract}
On the basis of our previous results concerning the breakdown of dilatation invariance we find that two large classes of models in quantum field theory may lead to the same observable quantities in spite of their different properties under scale transformation. Although the conditions of equivalence have long been known we emphasize their relevance in the new context of the dilatation transformation. The equivalence in question leads to the same physical masses, observable coupling constants, and, possibly, to the same energy-momentum tensors associated with the asymptotic fields in the two theories. It is suggested that the arbitrariness in the choice of an interpolating field is a key point for the equivalence of the two theories. Our analysis also underlines the difference between the notions of physical dimension and scale dimension of a field operator.
\end{abstract}

It is commonly accepted by now that dilatation invariance, in order to be a useful concept in particle physics, must be broken to a certain extent. It is also well known that the symmetry may be violated in any of the following ways:

(i) by explicitly introducing into the theory some breaking parameters which, incidentally, happen to be just the fundamental parameters of the theory, namely, masses and (dimensional) coupling constants (intrinsic breakdown);

(ii) by requiring that the vacuum be asymmetric under the dilatation transformation (spontaneous breakdown).

Of course, one also has to take into account possible combinations of intrinsic and spontaneous breakdown. In any case, however, in order to have a meaningful theory, the dynamics must produce massive observable particles.

We have remarked in previous paper $\mathrm{s}^{1,2}$ that the above requirement poses a consistency problem as far as spontaneous breakdown is concerned. Indeed, one begins with a Lagrangian which is fully invariant under the dilatation transformation and eventually ends up with massive observable particles thereby apparently violating the original invariance of the theory.

In Ref. 1 we have introduced the notion of "dimensional invariance" which, being simply an expression of the dimensional consistency of the theory, can never be violated. In Ref. 2 we have shown that dilatation invariance, with only spontaneous breakdown, may consistently lead to a physical mass and that the formal invariance of the theory is recovered, among the massive as ymptotic fields, in the form of dimensional invariance.

In this note we proceed one step further and inquire about the connection, if any, between intrinsic breakdown and spontaneous breakdown of scale invariance. More precisely we wish to find out if the two breaking techniques differ among themselves in some fundamental way or rather if they may lead to the same physics with the same observable particles. In the latter case we wish to establish the conditions of equivalence.

Our findings are that, in the framework of ordinary quantum field theory, there are well-known 